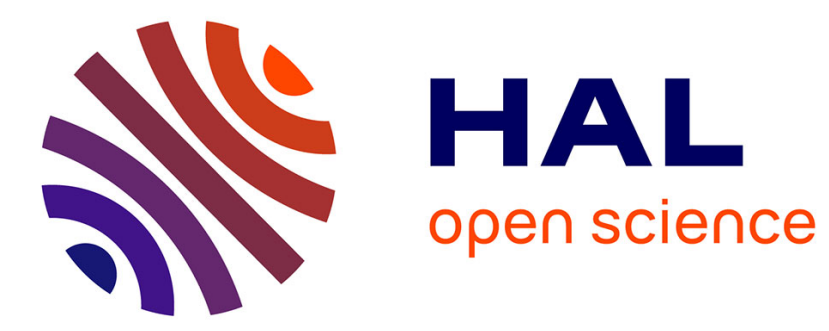

\title{
Non-trivial exponents in coarsening phenomena
}

B. Derrida

\section{To cite this version:}

B. Derrida. Non-trivial exponents in coarsening phenomena. Physica D: Nonlinear Phenomena, 1997, Lattice Dynamics, 103 (1-4), pp.466-477. 10.1016/S0167-2789(96)00278-3 . hal-03285617

\section{HAL Id: hal-03285617 https://hal.science/hal-03285617}

Submitted on 21 Jul 2021

HAL is a multi-disciplinary open access archive for the deposit and dissemination of scientific research documents, whether they are published or not. The documents may come from teaching and research institutions in France or abroad, or from public or private research centers.
L'archive ouverte pluridisciplinaire HAL, est destinée au dépôt et à la diffusion de documents scientifiques de niveau recherche, publiés ou non, émanant des établissements d'enseignement et de recherche français ou étrangers, des laboratoires publics ou privés. 


\title{
Non-trivial exponents in coarsening phenomena \\ B. Derrida \\ Laboratoire de Physique Statistique, ENS, 24 rue Lhomond, 75005 Paris, France
}

\begin{abstract}
One of the simplest examples of stochastic automata is the Glauber dynamics of ferromagnetic spin models such as Ising or Potts models. At zero temperature, if the initial condition is random, one observes a pattern of growing domains with a characteristic size which increases with time like $t^{1 / 2}$. In this self-similar regime, the fraction of spins which never flip up to time $t$ decreases like $t^{-\theta}$ where the exponent $\theta$ is non-trivial and depends both on the number $q$ of states of the Potts model and on the dimension of space. This exponent can be calculated exactly in one dimension.

Similar non-trivial exponents are also present in even simpler models of coarsening, where the dynamical rule is deterministic.
\end{abstract}

PACS: $02.50 ; 05.20$

\section{Introduction}

Domain growth and coarsening can be observed in a large variety of system [1], in particular in the Ising model of the $q$-state Potts model evolving according to zero temperature Glauber dynamics [2-10]. In the Ising case, the evolution rule is extremely simple: one starts with a random initial condition for a system of $N$ spins on a lattice (i.e. initially each spin $S_{i}(0)=+1$ or -1 with equal probability) and at each time step $\Delta t=1 / N$, a randomly chosen spin is updated: the spin is oriented in the direction of the majority of its neighbors and when there is no majority (i.e. the number of + neighbors equals the number of - neighbors), the updated value is chosen at random to be +1 or -1 with equal probability.

It is very easy to implement these dynamics: one calculates the local field $h_{i}(t)$ on the spin to be updated

$$
h_{i}(t)=\sum_{j \text { neighbor of } \mathrm{j}} S_{j}(t)
$$

and then one updates the spin by

$$
\begin{array}{ll}
\text { if } h_{i}(t) \neq 0, & S_{i}(t+\Delta t)=\operatorname{sign} h_{i}(t), \\
\text { if } h_{i}(t)=0, & S_{i}(t+\Delta t)=+1 \text { or }-1 \quad \text { with equal probability. }
\end{array}
$$

Fig. 1 shows the configurations at times $t=8,32,128,512,2048$, and 8192 of an Ising model on a square lattice of $400 \times 400$ spins. Clearly, the growth is self-similar and the size of domains $D \sim t^{1 / 2}$ as expected until it reaches the size of the lattice [1]. 

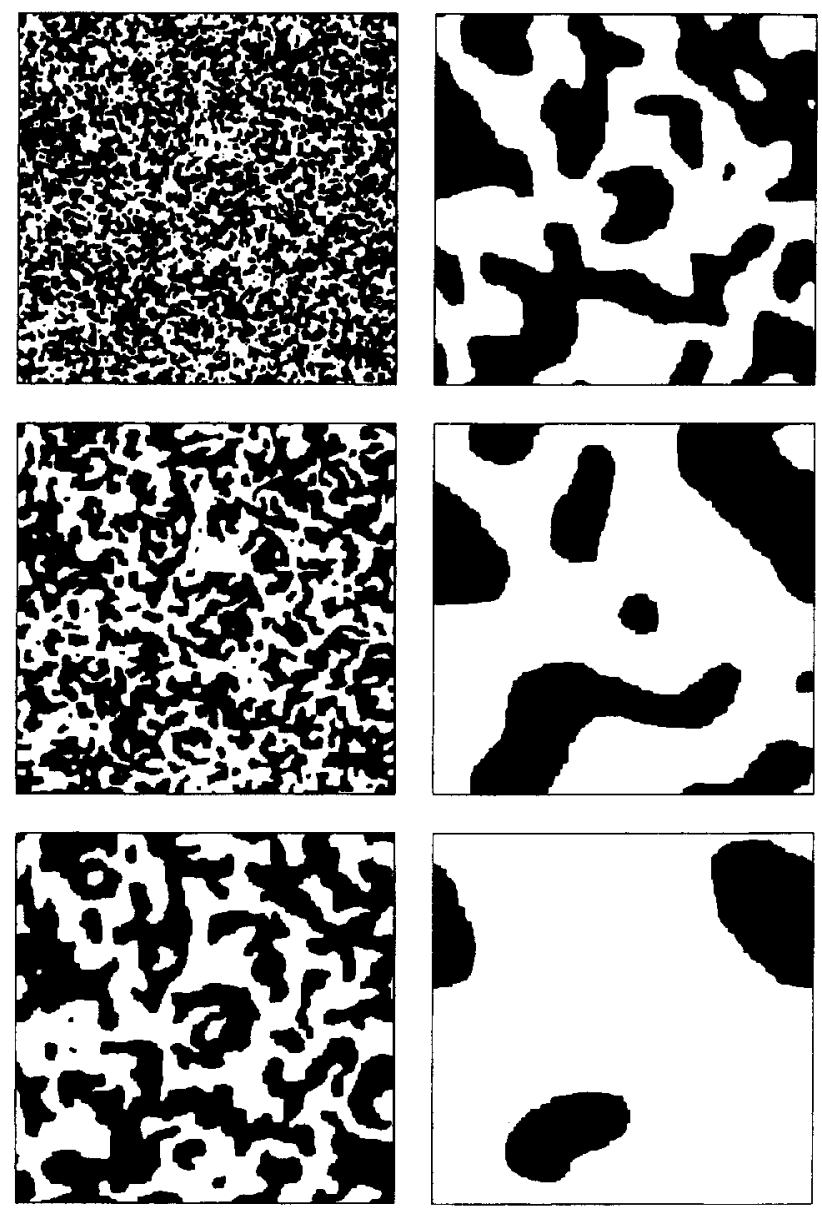

Fig. I. Configurations of an Ising model on a square lattice of $400 \times 400$ spins at times $t=8,32,128,2048$, and 8192 when the initial condition is random.

This self-similar growth can be observed in the power lay decay of various quantities. One can measure the energy $E(t)$ (normalized such that the ground state has zero energy),

$$
E(t)=\frac{1}{N} \sum_{i, j \text { neighbors }} \frac{1-S_{i}(t) S_{j}(t)}{2},
$$

the autocorrelation function $C(t)$,

$$
C(t)=\frac{1}{N} \sum_{i} S_{i}(t) S_{i}(0)
$$

or the fraction $F(t)$ of spins which have never flipped up to time $t$,

$$
F(t)=\frac{1}{N} \sum_{i} \prod_{0<\tau<t} \frac{1+S_{i}(\tau) S_{i}(0)}{2} .
$$

For all these quantities and many others, the power law decay (visible in Fig. 2 as a straight line in a log-log plot for square lattice of $1000 \times 1000$ spins) is the signature of a self-similar phenomenon. 


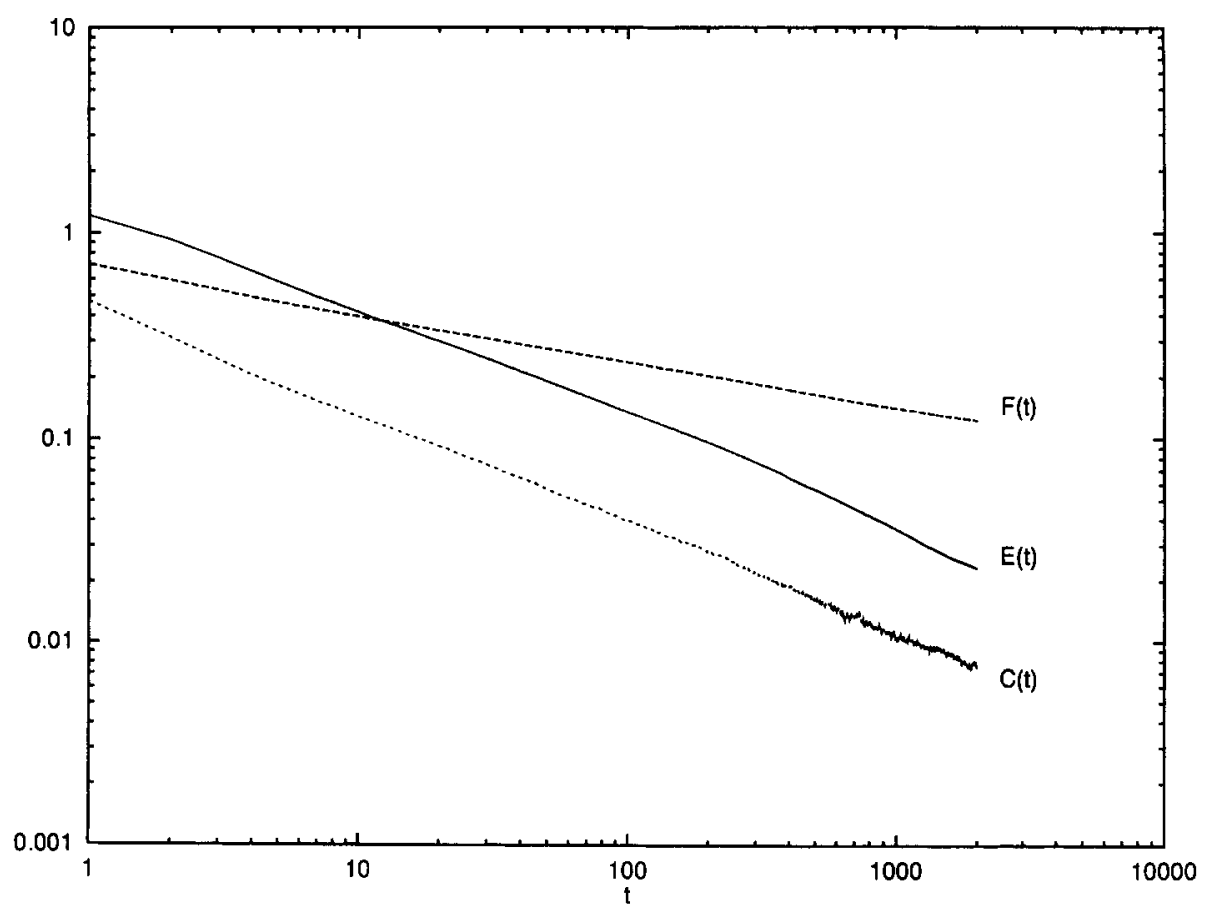

Fig. 2. The time dependence of the energy $E(t)$, of the autocorrelation $C(t)$ and of the fraction $F(t)$ of spins which never flip for an Ising model on a square lattice of $1000 \times 1000$ spins with nearest neighbor interactions.

The same zero temperature growth simulations can be repeated in many other systems. Unless the growth process is slowed down or completely stopped by blocked configurations (like in the $q$-state Potts model on a square lattice with nearest neighbor interactions $[3,5,10])$, one observes a self-similar growth with a variety of patterns and power law decays of $E(t), C(t)$ or $F(t)$. For the $q$-state Potts model with nearest and next nearest neighbor on a square lattice, there is no blocking and one observes growing patterns as in Fig. 3 for $q=\infty$ (the case $q=\infty$ has been studied in the context of soap froths [11,12] and polycrystals [13]). Fig. 4 shows a measure of the energy $E(t)$, of the autocorrelation function $C(t)$ and of the fraction $F(t)$ of spins which do not flip up to time $t$ for a square lattice of $1000 \times 1000$ sites with nearest and next nearest neighbor interactions for $q=7$, these quantities being defined for the $q$-state Potts model by

$$
\begin{aligned}
& E(t)=\frac{1}{N} \sum_{i, j} 1-\delta_{s_{i}(t), s_{j}(t)}, \quad C(t)=-\frac{1}{q}+\frac{1}{N} \sum_{i} \delta_{s_{i}(t), s_{i}(0)} ; \\
& F(t)=\frac{1}{N} \sum_{i} \prod_{0<\tau<t} \delta_{s_{i}(\tau), s_{i}(0)} .
\end{aligned}
$$

For the $q$-state Potts model as for the Ising case, one observes power law decays characterized by exponents $[9,10]$ which in general depend on $q$. Only, the energy [5,14] seems to always decay with the exponent $\frac{1}{2}$. Under the assumption that the domain boundaries are not fractal this exponent is the same as the one describing the domain size $D(t) \sim t^{1 / 2}$ since $E(t) \sim D^{-1}(t)$. The exact values of the other exponents (decay of $C(t)$ or $F(t)$ ) are still unknown in general dimension with the exception of the one-dimensional case to be discussed below and of the two-dimensional Ising model for which it has been conjectured [4] that $C(t) \sim t^{-5 / 8}$. 

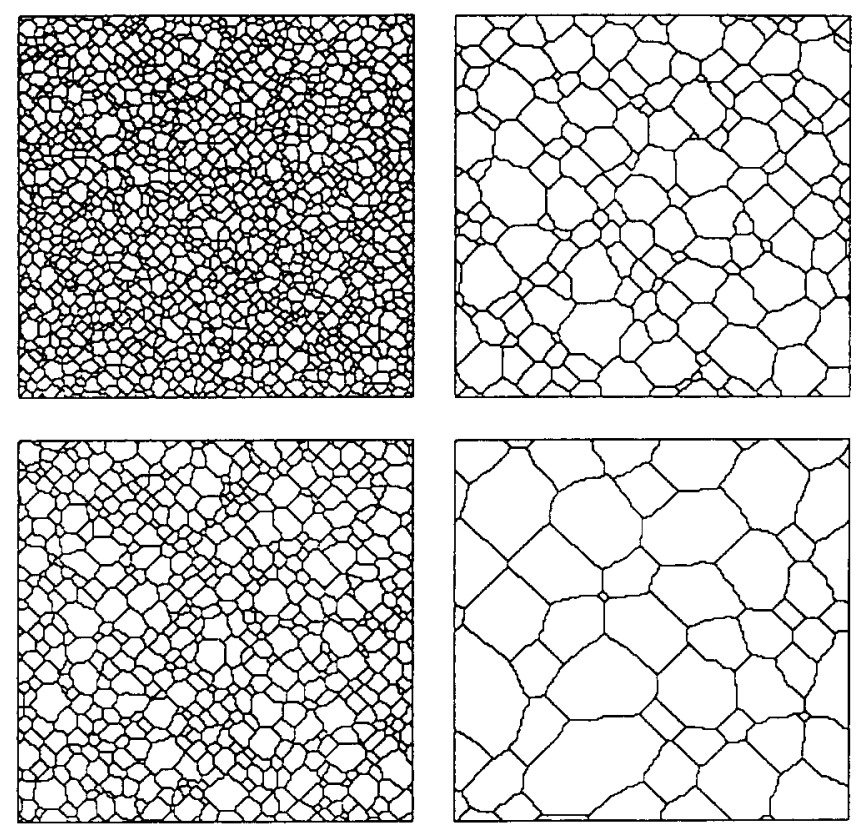

Fig. 3. Configurations of a Potts model (for $q=\infty$ ) on a square lattice of $200 \times 200$ spins with nearest and next nearest neighbor interactions at times $t=20,80,320$ and 1280 when the initial condition is random (when $q=\infty$ all the spins have initially different culors).

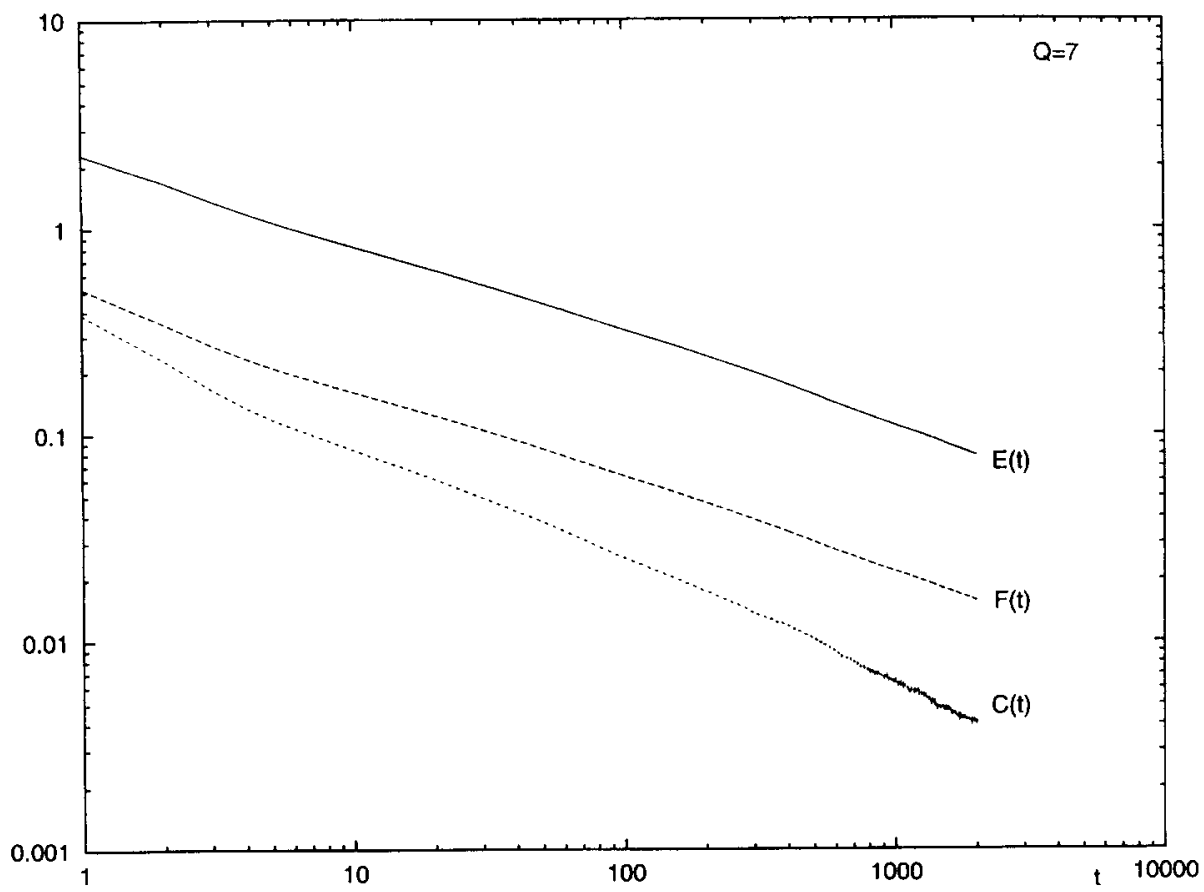

Fig. 4. The time dependence of the energy $E(t)$, of the autocorrelation $C(t)$ and of the fraction $F(t)$ of spins which never flip for a Potts model $(q=7)$ on a square lattice of $1000 \times 1000$ spins with nearest and next nearest neighbor interactions. 


\section{The one-dimensional case}

In one dimension, the zero temperature Glauber dynamics is equivalent to that of a voter model: during each infinitesimal time interval $\Delta t$, each spin $S_{i}(t)$ has a probability $\Delta t$ of being updated and when this happens, it takes with probability $\frac{1}{2}$ the value of its left neighbor and with probability $\frac{1}{2}$ the value of its right neighbor;

$$
S_{i}(t+\Delta t)= \begin{cases}S_{i}(t) & \text { with probability } 1-\Delta t \\ S_{i-1}(t) & \text { with probability } \frac{1}{2} \Delta t \\ S_{i+1}(t) & \text { with probability } \frac{1}{2} \Delta t .\end{cases}
$$

Because of this equivalence with the voter model, random walks methods can be used and a number of quantities can be calculated exactly $[15,19]$.

In the Ising case, for a random initial condition, one can obtain exact expressions of several quantities. The dynamical rule (7) implies for example that the two-point correlation function for $i<j$ evolves according to

$$
\frac{\mathrm{d}\left\langle S_{i} S_{j}\right\rangle}{\mathrm{d} t}=\frac{\left\langle S_{i+1} S_{j}\right\rangle+\left\langle S_{i-1} S_{j}\right\rangle+\left\langle S_{i} S_{j-1}\right\rangle+\left\langle S_{i} S_{j+1}\right\rangle-4\left\langle S_{i} S_{j}\right\rangle}{2}
$$

and for a random initial condition $\left(\left\langle S_{i}(0) S_{j}(0)\right\rangle=\delta_{i, j}\right)$, one obtain the following exact expression for the equal time correlation function valid when $i<j$ :

$$
\left\langle S_{i}(t) S_{j}(t)\right\rangle=\frac{1}{2 \pi} \int_{0}^{2 \pi} \mathrm{d} \theta \sin [(j-i) \theta] \sin \theta \frac{1-\mathrm{e}^{-2 t(1-\cos \theta)}}{1-\cos \theta} .
$$

This gives in particular for the energy $E(t)=\frac{1}{2}\left[1-\left\langle S_{i}(t) S_{i+1}(t)\right\rangle\right]$ :

$$
E(t)=\frac{1}{4 \pi} \int_{0}^{2 \pi} \mathrm{d} \theta(1+\cos \theta) \mathrm{e}^{-2 t(1-\cos \theta)} .
$$

One can also calculate the autocorrelation function (for a random initial condition)

$$
C(t)=\left\langle S_{i}(t) S_{i}(0)\right\rangle=\frac{1}{2 \pi} \int_{\delta}^{2 \pi} \mathrm{d} \theta \mathrm{e}^{-t(1-\cos \theta)},
$$

and in the long time limit, one obtains

$$
E(t) \simeq \frac{1}{\sqrt{2}} C(t) \simeq \frac{1}{2 \sqrt{\pi t}} .
$$

Thus the exponents characterizing the decay of the energy and of the autocorrelation function are both $\frac{1}{2}$. From (8), one can also show that a scaling is valid in the long time limit

$$
\left\langle S_{i}(t) S_{j}(t)\right\rangle \simeq f\left(\frac{|j-i|}{\sqrt{t}}\right),
$$

where

$$
f(x)=\frac{1}{\sqrt{\pi}} \int^{\infty} \mathrm{e}^{-u^{2} / 4} \mathrm{~d} u .
$$


There exists a characteristic length $L(t)=\sqrt{t}$ and, as $t \rightarrow \infty$, the correlation function becomes a function of the single variable $|j-i| / L(t)$.

Another property which can be calculated exactly is the autocorrelation at two different times. For example for $t \gg 1$ and $t^{\prime}-t \gg 1$, one finds that it becomes a function of the ratio $t / t^{\prime}$ only:

$$
\left\langle S_{i}\left(t^{\prime}\right) S_{i}(t)\right\rangle \simeq 1-\frac{2}{\pi} \tan ^{-1} \sqrt{\frac{t^{\prime}-t}{2 t}} .
$$

All these calculations can be easily generalized to the one-dimensional $q$-state Potts model. The dynamics are still governed by ( 7$)$, the only difference being that the initial values $S_{i}(0)$ are chosen at random among $q$ colors. As in the Ising case, exact expressions of various quantities can be obtained using random walks. For an uncorrelated initial condition (i.e. such that $\operatorname{Prob}\left(S_{i}(0)=S_{j}(0)\right)=1 / q$ for $i \neq j$ ), one obtains in the long time limit the equal time correlation function

$$
\left\langle\delta_{S_{i}(t) . S_{j}(t)}-\frac{1}{q}\right\rangle \simeq \frac{q-1}{q} \frac{1}{\sqrt{\pi}} \int_{|j-i| / \sqrt{t}}^{\infty} \mathrm{e}^{-u^{2} / 4} \mathrm{~d} u
$$

and consequently the energy (5)

$$
E(t) \simeq \frac{q-1}{q} \frac{1}{\sqrt{\pi t}}
$$

whereas the autocorrelation function $C(t)$ is given by

$$
C(t)=\left\langle\delta_{S_{i}(t), S_{i}(0)}-\frac{1}{q}\right\rangle=\frac{q-1}{q} \frac{1}{2 \pi} \int_{0}^{2 \pi} \mathrm{d} \theta \mathrm{e}^{-t(1-\cos \theta)} \simeq \frac{q-1}{q} \frac{1}{\sqrt{2 \pi t}} .
$$

As in the Ising case one finds that $E(t)$ and $C(t)$ decay with an exponent $\frac{1}{2}$ for all values of $q$.

Recently, it was observed [6] that even in one dimension, the fraction $F(t)$ of spins which never flip up to time $t$ in a zero temperature dynamics decays according to a power law with a $q$-dependent exponent

$$
F(t) \sim t^{-\theta(q)} .
$$

The exponent $\theta(q)$ is very easy to measure in a Monte Carlo calculation (see Fig. 5 and the corresponding Fortran program in Fig. 6). One takes a large enough one-dimensional lattice (here a lattice of 1000000 sites) and counts the number of spins which never flip. These simulations lead to the following estimates for the exponent: $\theta(2) \simeq 0.376$, $\theta(3) \simeq 0.53, \theta(5) \simeq 0.70, \theta(10) \simeq 0.82$.

Recently, these results were confirmed by an exact expression [20] of the exponent $\theta(q)$

$$
\theta(q)=-\frac{1}{8}+\frac{2}{\pi^{2}}\left[\cos ^{-1}\left(\frac{2-q}{\sqrt{2} q}\right)\right]^{2} .
$$

which gives $\theta(2) \simeq 0.375, \theta(3) \simeq 0.5379508 \ldots, \theta(5) \simeq 0.6928365 \ldots, \theta(10) \simeq 0.8310356 \ldots$ in agreement with Monte Carlo [6,7] and finite size scaling estimates [21].

It would be too long to repeat here the whole derivation of (17). We just give two important ingredients of the solution $[20,22]$. If one considers an infinite chain with a random initial condition, $F(t)$ is the probability that the spin at the origin (or in fact any other spin) never flips up to time $t$. If the spin at the origin never flips, this decouples its two sides and if $f(t)$ is the probability that the spin at the origin of a semi infinite chain never flips, one has

$$
F(t)=f^{2}(t)
$$




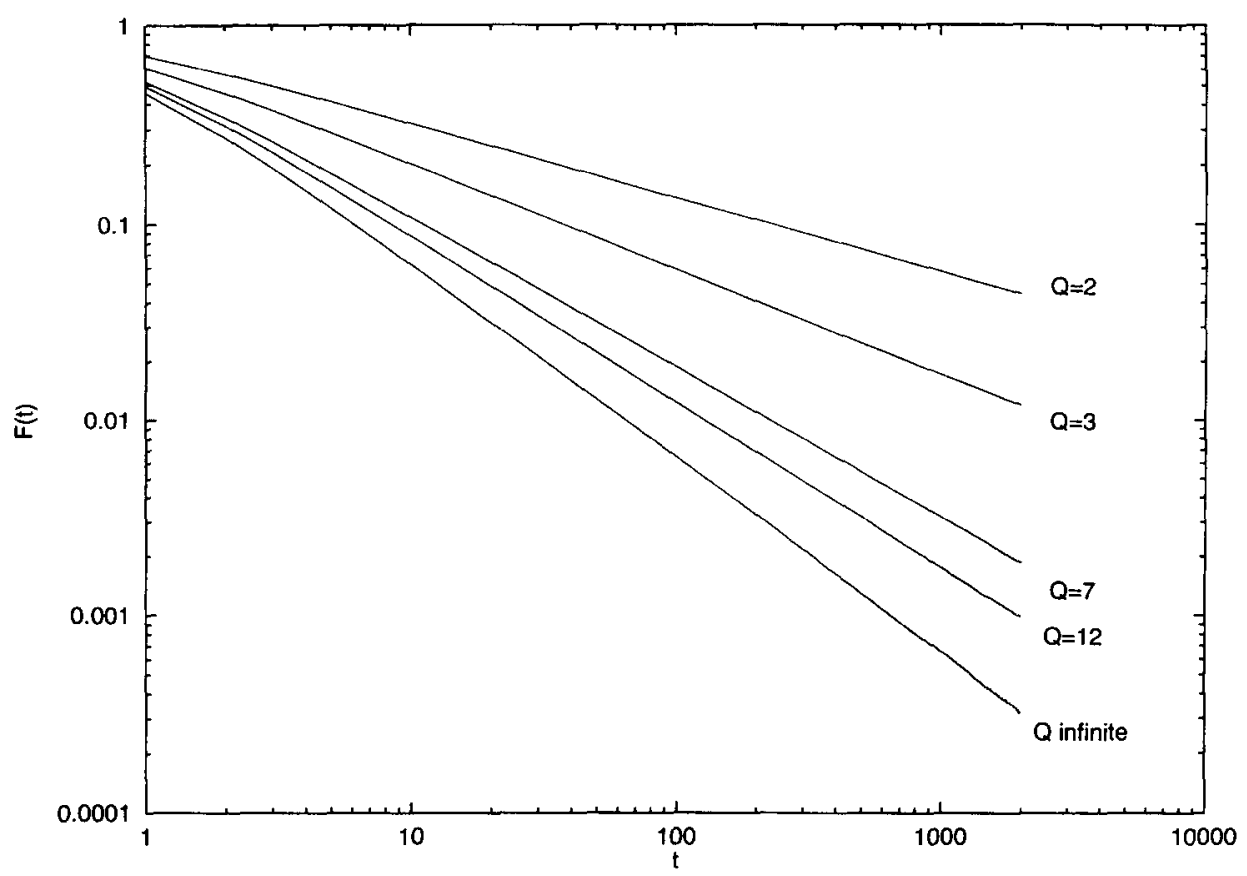

Fig. 5. The fraction $F(t)$ of spins which never flip up to time $t$ for a one dimensional $q$-state Potts model on a lattice of $10^{6}$ sites. One observes a power law decay with a $q$-dependent exponent $\theta(q)$.

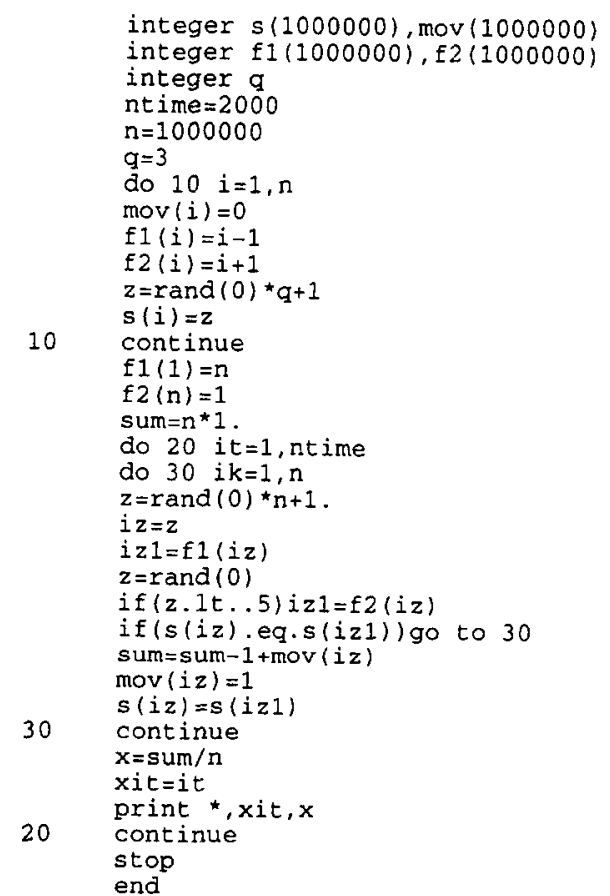

Fig. 6. A simple program (not an optimized version) to generate the data of Fig. 5. 
as the two sides of the origin are independent. Now, $f(t)$ can be calculated as

$$
f(t)=\lim _{N \rightarrow \infty} \phi\left(\tau_{1}, \tau_{2}, \ldots, \tau_{N}\right),
$$

where $\phi\left(\tau_{1}, \tau_{2}, \ldots, \tau_{N}\right)$ is the probability that the spin at the origin takes the same value at times $\tau_{1}, \tau_{2}, \ldots, \tau_{N}$. Clearly, if the times $\tau_{1}, \tau_{2} \ldots \tau_{N}$ become dense in the time interval $0, t$ (for example by choosing $\tau_{i}=t_{i} / N$ ), one gets $f(t)$. It turns out that it is not difficult to calculate exactly $\phi\left(\tau_{i}, \tau_{j}\right)$ for $\tau_{i}<\tau_{j}$

$$
\phi\left(\tau_{i}, \tau_{j}\right)=1-\frac{q-1}{q} c\left(\tau_{i}, \tau_{j}\right) .
$$

$c\left(\tau_{i}, \tau_{j}\right)$ is the probability that two random walkers on a semi-infinite lattice starting at times $-\tau_{i}$ and $-\tau_{j}$ do not meet until time 0 . One can calculate $c\left(\tau_{i}, \tau_{j}\right)$ exactly and for large $\tau_{i}$ and $\tau_{j}$, with $\tau_{i}<\tau_{j}$, one obtains

$$
c\left(\tau_{i}, \tau_{j}\right) \simeq \frac{4}{\pi} \tan ^{-1} \sqrt{\frac{\tau_{j}}{\tau_{i}}}-1 .
$$

The main step which led to the exact determination of the exponent $\theta$ was to realize that all the $\phi\left(\tau_{1}, \tau_{2}, \ldots, \tau_{N}\right)$ can be expressed in terms of the same matrix $c$. For example for $\tau_{1}<\tau_{2}<\tau_{3}$

$$
\phi\left(\tau_{1}, \tau_{2}, \tau_{3}\right)=1-\frac{q-1}{q^{2}}\left[c\left(\tau_{1}, \tau_{2}\right)+c\left(\tau_{2}, \tau_{3}\right)\right]-\frac{(q-1)^{2}}{q^{2}} c\left(\tau_{1}, \tau_{3}\right),
$$

and for $\tau_{1}<\tau_{2}<\tau_{3}<\tau_{4}$

$$
\begin{aligned}
\phi\left(\tau_{1}, \tau_{2}, \tau_{3}, \tau_{4}\right)= & 1-\frac{q-1}{q^{2}}\left[c\left(\tau_{1}, \tau_{2}\right)+c\left(\tau_{2}, \tau_{3}\right)+c\left(\tau_{3}, \tau_{4}\right)\right]-\frac{(q-1)^{2}}{q^{2}} c\left(\tau_{1}, \tau_{4}\right) \\
& +\frac{(q-1)^{2}}{q^{3}}\left[c\left(\tau_{1}, \tau_{2}\right) c\left(\tau_{3}, \tau_{4}\right)+c\left(\tau_{1}, \tau_{4}\right) c\left(\tau_{2}, \tau_{3}\right)-c\left(\tau_{1}, \tau_{3}\right) c\left(\tau_{2}, \tau_{4}\right)\right] .
\end{aligned}
$$

Writing the general expression of $\phi\left(\tau_{1}, \tau_{2}, \ldots, \tau_{N}\right)$ and taking the limit $N \rightarrow \infty$ leads $[20,22]$ to (17).

Remark. The same relation between two-point functions and higher correlations exists for equal time correlations. If $\Phi\left(x_{1}, x_{2}, \ldots, x_{N}\right)$ is the probability that $S_{x_{1}}(t)=S_{x_{2}}(t)=\cdots=S_{x_{N}}(t)$ in the zero temperature dynamics of the $1 d$ Potts model, one can show that all the $\Phi$ can be expressed in terms of a single matrix $C$, the element $C(x, y)$ of which is the probability that two random walkers starting at positions $x$ and $y$ at time 0 do not meet up to time $t$. In the long time limit, one can show that

$$
C(x, y) \simeq \frac{1}{\sqrt{\pi}} \int_{0}^{|x-y| / \sqrt{t}} \mathrm{e}^{-u^{2} / 4} \mathrm{~d} u
$$

and the expression of the $\Phi$ in terms of the matrix $C$ are the same as above. For $x_{1}<x_{2}$,

$$
\Phi\left(x_{1}, x_{2}\right)=1-\frac{q-1}{q} C\left(x_{1}, x_{2}\right)
$$

for $x_{1}<x_{2}<x_{3}$,

$$
\Phi\left(x_{1}, x_{2}, x_{3}\right)=1-\frac{q-1}{q^{2}}\left[C\left(x_{1}, x_{2}\right)+C\left(x_{2}, x_{3}\right)\right]-\frac{(q-1)^{2}}{q^{2}} C\left(x_{1}, x_{3}\right),
$$

and for $x_{1}<x_{2}<x_{3}<x_{4}$, 


$$
\begin{aligned}
\Phi\left(x_{1}, x_{2}, x_{3}, x_{4}\right)= & 1-\frac{q-1}{q^{2}}\left[C\left(x_{1}, x_{2}\right)+C\left(x_{2}, x_{3}\right)+C\left(x_{3}, x_{4}\right)\right]-\frac{(q-1)^{2}}{q^{2}} C\left(x_{1}, x_{4}\right) \\
& +\frac{(q-1)^{2}}{q^{3}}\left[C\left(x_{1}, x_{2}\right) C\left(x_{3}, x_{4}\right)+C\left(x_{1}, x_{4}\right) C\left(x_{2}, x_{3}\right)-C\left(x_{1}, x_{3}\right) C\left(x_{2}, x_{4}\right)\right] .
\end{aligned}
$$

By taking the limit of a large number $N$ of points when the points become dense (on the lattice one can choose consecutive points), it is possible [34] to use the exact expressions of the $\Phi$ to determine the distribution of domain sizes for the general $q$-state Potts model.

\section{A deterministic growth model}

There are other one-dimensional models which exhibit coarsening phenomena. One of them is the one-dimensional Ginzburg-Landau equation [29]. A configuration of the system at time $t$ is represented by a function $F(x, t)$ and it evolves deterministically according to

$$
\frac{\partial F}{\partial t}=\frac{\partial^{2} F}{\partial x^{2}}+F-F^{3}
$$

Regions where $F$ is positive correspond to the + phase whereas $F$ is negative in the - phase. A major difference with the zero temperature dynamics of the Ising chain discussed in Section 2 is that (18) is deterministic and randomness is only present in the initial condition. If one starts with a random initial condition where $-1 \leq F(x, 0) \leq 1$, one observes a coarsening phenomenon. In the long time limit, $F(x, t)=1$ or -1 almost everywhere except at domain walls. As the domains grow, the typical size of the domains becomes much larger than the width of domain walls and on a scale where the average domain size is one, domain walls look like points. In this limit, the dynamics becomes very simple: the closest pair of walls move together and annihilate while the other walls hardly move at all and the system coarsens by eliminating the smallest domains [29,30].

So one is left with a very simple model of domain growth. One starts with segments of random lengths and alternating signs along the line. At each step of the evolution, the sign of the shortest domain is reversed and consequently, a new domain is formed composed of this shortest domain and of its two neighbors. In the scaling limit, one can calculate exactly the distribution of domain sizes $[29,31,32]$. One can also calculate the exponent $\lambda$ which governs the decay of the autocorrelation function

$$
\langle F(x, t) F(x, 0)\rangle \sim L^{\lambda-1},
$$

where $L$ is the characteristic size of the domains at time $t$. The exponent $\lambda$ is exactly given [33] by the zero of the following transcendental equation:

$$
\int_{0}^{\infty} q^{\lambda-2} \mathrm{e}^{-q}\left[\left(1-q-\mathrm{e}^{-q}\right) \mathrm{e}^{r(q)}+q^{2}(1-\lambda) \mathrm{e}^{-r(q)}\right] \mathrm{d} q=0,
$$

where

$$
r(p)=\int_{p}^{\infty} \frac{\mathrm{e}^{-q}}{q} \mathrm{~d} q+\log p
$$

leading to

$$
\lambda \simeq 0.3993835 \ldots
$$


Similarly, one can show [32] that the fraction $F(t)$ of the line which has never flipped decays like

$$
F(t) \sim L^{\beta-1},
$$

where the exponent $\beta=0.82492412 \ldots$ is solution of another transcendental equation

$$
\int_{0}^{\infty} q^{\beta-2} \mathrm{e}^{-q}\left[\left(1-q-\mathrm{e}^{-q}\right) \mathrm{e}^{r(q)}+2 q(1-\beta)+q^{2}(1-\beta) \mathrm{e}^{-r(q)}\right] \mathrm{d} q=0 .
$$

These results $\left(C(t) \sim L^{-0.600616 \ldots}\right.$ and $\left.F(t) \sim L^{-0.17507587 \ldots}\right)$ valid for the deterministic dynamics (18) have to be compared with results discussed in Section 2, namely that for the zero temperature Glauber dynamics $C(t) \sim L^{-1}$ and $F(t) \sim L^{-0.75}$. The main difference between the two growth mechanisms is that one is deterministic and the other one is stochastic. This seems to contradict the idea that noise is irrelevant in coarsening [1]. A possible explanation of that contradiction is that one dimension is special because $T=0$ is also the ordering temperature.

\section{Conclusion}

We have seen that non-trivial exponents characterize the decay of unequal time correlations in zero temperature coarsening phenomena. For the one-dimensional $q$-state Potts model, the fraction $F(t)$ of spins which never flip decays $(16,17)$ like a power law with an exponent $\theta$ which depends on $q$.

The first question one can ask is whether one could consider different quantities characteristic of this onedimensional growth problem, which would decay with other non-trivial exponents.

In dimension higher than 1 , the exponent $\theta$ has been measured $[6,7,10]$ in Monte Carlo simulations. It would be interesting to know whether other approaches could be implemented to predict this exponent, and in particular to determine the upper critical dimension, if there is one.

Another interesting issue is that of thermal noise. If $F(t)$ is defined as the number of spins which never flip, it certainly will decay exponentially at any non-zero temperature. On the other hand, when one observes a coarsening phenomenon at low temperature, one can talk of domains of the two ordered phases of an Ising model (at least when the size of the domains is much larger than the bulk correlation length) and so one can try to measure the fraction of space which remains always in the same phase. One difficulty which, to author's knowledge, remains to be solved is to have a good definition of this fraction, in order to be able to measure it in a simulation.

Also, it would be interesting to check whether in dimension larger than 1, noise is irrelevant [1] and if the zero temperature Glauber dynamics gives the same exponent as the Landau-Ginzburg equation.

Lastly, one can interpret the zero temperature dynamics of the $q$-state Potts model as a reaction diffusion problem [23-26,28]. If the domain walls are represented by $A$ particles, these particles diffuse on the one-dimensional lattice, and whenever two particles $A$ meet on the same lattice site, they instantaneously react according to:

$$
\begin{aligned}
A+A & \rightarrow A \quad \text { with probability }(q-2) /(q-1), \\
& \rightarrow 0 \quad \text { with probability } 1 /(q-1) .
\end{aligned}
$$

So when two particles meet, they either aggregate with probability $(q-2) /(q-1)$ to form a new particle A or they annihilate with probability $1 /(q-1)$.

All the exactly known results on the zero temperature dynamics of the $1 d$ Potts model can be reinterpreted in terms of reaction diffusion models of the domain walls. For example, the fact that all the equal time correlations $\Phi\left(x_{1}, x_{2}, \ldots, x_{N}\right)$ can be determined exactly for the dynamics of the $1 d$ Potts model (as discussed at the very 
end of Section 2) implies that one can calculate arbitrary correlation functions for the reaction diffusion model. If $\Psi\left(x_{1}, x_{2}, \ldots, x_{k}\right)$ is the probability of finding particles $A$ at positions $x_{1}, x_{2}, \ldots, x_{k}$, one has

$$
\Psi\left(x_{1}, x_{2}, \ldots, x_{k}\right)=\text { Probability }\left\{S_{x_{1}}(t) \neq S_{x_{1}+1}(t) ; S_{x_{2}}(t) \neq S_{x_{2}+1}(t) ; \ldots S_{x_{k}}(t) \neq S_{x_{k}+1}(t)\right\}
$$

and this can be obtained from the $\Phi$. For example

$$
\begin{aligned}
& \Psi\left(x_{1}\right)=1-\Phi\left(x_{1}, x_{1}+1\right), \\
& \Psi\left(x_{1}, x_{2}\right)=1-\Phi\left(x_{1}, x_{1}+1\right)-\Phi\left(x_{2}, x_{2}+1\right)+\Phi\left(x_{1}, x_{1}+1, x_{2}, x_{2}+1\right) .
\end{aligned}
$$

In particular, in the long time limit, one recovers from (15) that the density of particles $\rho_{A}=\psi\left(x_{1}\right)$

$$
\rho_{A} \simeq \frac{q-1}{q} \frac{1}{\sqrt{\pi t}}
$$

decays as $t^{-1 / 2}$ as expected $[23,25]$.

\section{Acknowledgements}

This lecture has been inspired by very pleasant and stimulating interactions with A.J. Bray, D. Dhar, M. Droz, H. Flyvbjerg, C. Godréche, V. Hakim, P.M.C. de Oliveira, V. Pasquier, D. Stauffer, I. Yekutieli and R. Zeitak.

\section{References}

[1] A.J. Bray, Adv. Phys. 43 (1994) 357.

[2] R.J. Glauber, J. Math. Phys. 4 (1963) 294.

[3] P.S. Sahni, D.J. Srolovitz, G.S. Grest, M.P. Anderson and S.A. Safran, Phys. Rev. B 28 (1983) 2705.

[4] D.S. Fisher and D.A. Huse, Phys. Rev. B 38 (1988) 373.

[5] G.S. Grest, D.J. Srolovitz and M.P. Anderson, Phys. Rev. B 38 (1988) 4752.

[6] B. Derrida, A.J. Bray and C. Godreche, J. Phys. A 27 (1994) L357.

[7] D. Stauffer, J. Phys. A 27 (1994) 5029.

[8] S.N. Majumdar and D.A. Huse, Phys. Rev. E 52 (1995) 270.

[9] C. Sire and S.N. Majumdar, Phys. Rev. E 52 (1995) 244.

[10] B. Derrida, P.M.C. de Oliveira and D. Stauffer, Physica A 224 (1996) 604.

[11] H. Flyvbjerg, Phys. Rev. E 47 (1988) 4037.

[12] J.A. Glazier, S.P. Gross and J. Stavans, Phys. Rev A 36 (1987) 306

[13] M.P. Anderson, G.S. Grest, D.J. Srolovitz and S.A. Safran, Philos. Mag. 59 (1989) 293.

[14] M. Lau, C. Dasgupta and O.T. Valls, Phys. Rev B 38 (1988) 9024.

[15] Z. Rácz, Phys. Rev. Lett. 55 (1985) 1707.

[16] A.J. Bray, J. Phys. A 23 (1990) L67.

[17] J.G. Amar and F. Family, Phys. Rev. A 41 (1990) 3258.

[18] B. Derrida, C. Godreche and I. Yekutieli, Phys. Rev. A 44 (1991) 6241.

[19] V. Privman, J. Statist. Phys. 69 (1992) 629.

[20] B. Derrida, V. Hakim and V. Pasquier, Phys. Rev. Lett. 75 (1995) 751.

[21] B. Derrida, J. Phys. A 28 (1995) 1481.

[22] B. Derrida, V. Hakim and V. Pasquier, J. Stat. Phys. 85 (1996) 763.

[23] K. Kang and S. Redner, Phys. Rev. Lett. 52 (1984) 955; Phys. Rev. A 32 (1985) 435.

[24] D. ben-Avraham, M.A. Burschka and C.R. Doering, J. Statist. Phys. 60 (1990) 695.

[25] S. Cornell, M. Droz and B. Chopard, Physica A 188 (1992) 322.

[26] B.P. Lee, J. Phys. A 27 (1994) 2633.

[27] P.L. Krapivsky, E. ben-Naim and S. Redner, Phys. Rev. E 50 (1994) 2474.

[28] J.L. Cardy, J. Phys. A 28 (1995) L19. 
[29] T. Nagai and K. Kawasaki, Physica A 134 (1986) 483.

[30] J. Carr and R. Pego, Proc. Roy. Soc. London A 436 (1992) 569.

[31] A.D. Rutenberg and A.J. Bray, Phys. Rev. E 50 (1994) 1900.

[32] A.J. Bray, B. Derrida and C. Godreche, Europhys. Lett. 27 (1994) 175.

[33] A.J. Bray, B. Derrida, Phys. Rev. E 51 (1995) 1633.

[34] B. Derrida and R. Zeitak, Phys. Rev. E 54 (1996) 2513. 